\section{What use do patients with headache make of the internet for medical information?}

\section{Andrew J. Larner}

Sir:

Internet websites containing medical information may be accessed with a suitably connected computer without recourse to a doctor. Information thus acquired may shape health beliefs and expectations, appropriately or otherwise. This study asked what use patients with headache make of internet medical websites.

Consecutive new referrals attending general neurology outpatient clinics at two district general hospitals in north-west England, seen during the first quarter of 2001-2004 inclusive, were asked about their access to and use of the internet for obtaining medical information [1]. Prior studies show headache to account for approximately $20 \%$ of such referrals [2].

Of 854 new patients seen, 154 (18\%) had headache. Of these, 79 $(51 \%)$ had home access to the internet. Sixteen had accessed websites with medical information prior to consultation, of whom 14 (9\%) had accessed information they believed relevant to their headache problem. Only one patient volunteered this information, along with material downloaded from the internet, prior to specific questioning.

Seven patients made self-diagnoses based on information obtained from internet searches, but in six this differed from the neurologist's diagnosis. Four patients with chronic tension-type headache self-diagnosed migraine, cerebral hypotension, osteoarthritis and temporomandibular joint dysfunction, respectively. A selfdiagnosis of antiphospholipid antibody syndrome was made by a patient with migraine without aura; and of cluster headache or acoustic neuroma by a patient with migraine with aura. Six patients reported no extra information gained from internet searches and/or were confused by the amount of material available.

Although internet searching may contribute positively to the physicianpatient relationship [3], this study suggests that unstructured internet searching by patients with headache may not be helpful. Internet-based information on headache, though extensive, has been judged poorly organised [4], which may have contributed to the findings of this study.
There are various websites appropriate to headache to which patients may be directed [5].

Acknowledgements Thanks to Julie Lyon and Cheryl Whittingham-Jones for help with data retrieval.

\section{References}

1. Larner AJ (2002) Use of internet medical websites and NHS Direct by neurology outpatients before consultation. Int J Clin Pract 56:219-221

2. Larner AJ (2003) NHS Direct for headache. J Neurol Neurosurg Psychiatry 74:1698

3. Murray E, Lo B, Pollack L et al (2003) The impact of health information on the internet on the physician - patient relationship: patient perceptions. Arch Intern Med 163:1727-1734

4. Peroutka SJ (2001) Analysis of internet sites for headache. Cephalalgia 21:20-24

5. Martelletti P (2000) On the web. J Headache Pain 1:133-134

A.J. Larner (可)

Walton Centre for Neurology

and Neurosurgery,

Lower Lane, Fazakerley,

Liverpool L9 7LJ, UK

e-mail: a.larner@thewaltoncentre.nhs.uk Fax: +44-151-5295513 